\title{
BMJ Open Variability in hospital treatment costs: a time-driven activity-based costing approach for early-stage invasive breast cancer patients
}

\author{
Erin Roman (10 , ${ }^{1}$ Brecht Cardoen, ${ }^{1,2}$ Jan Decloedt, ${ }^{3}$ Filip Roodhooft ${ }^{2,4}$
}

To cite: Roman E, Cardoen B, Decloedt J, et al. Variability in hospital treatment costs: a time-driven activitybased costing approach for early-stage invasive breast cancer patients. BMJ Open 2020;10:e035389. doi:10.1136/ bmjopen-2019-035389

- Prepublication history and additional material for this paper are available online. To view these files, please visit the journal online (http://dx.doi. org/10.1136/bmjopen-2019035389).

Received 31 October 2019 Revised 04 March 2020 Accepted 29 May 2020

\section{Check for updates}

C Author(s) (or their employer(s)) 2020. Re-use permitted under CC BY-NC. No commercial re-use. See rights and permissions. Published by BMJ.

${ }^{1}$ Health Care Management Centre, Vlerick Business School, Gent, Belgium

${ }^{2}$ Faculty of Economics and Business (FEB), KU Leuven, Leuven, Flanders, Belgium ${ }^{3}$ Breast Clinic, AZ Sint-Blasius, Dendermonde, Oost-Vlaanderen, Belgium

${ }^{4}$ Accounting and Finance, Vlerick Business School, Gent, Belgium

Correspondence to

Erin Roman;

erin.roman@vlerick.com

\section{ABSTRACT}

Objectives Using a standardised diagnostic and generic treatment path for breast cancer, and the molecular subtype perspective, we aim to measure the impact of several patient and disease characteristics on the overall treatment cost for patients. Additionally, we aim to generate insights into the drivers of cost variability within one medical domain.

Design, setting and participants We conducted a retrospective study at a breast clinic in Belgium. We used 14 anonymous patient files for conducting our analysis.

Results Significant cost variations within each molecular subtype and across molecular subtypes were found. For the luminal A classification, the cost differential amounts to roughly $166 \%$, with the greatest treatment cost amounting to US\$29 780 relative to US\$11 208 for a patient requiring fewer medical activities. The major driver for these cost variations relates to disease characteristics. For the luminal B classification, a cost difference of roughly $242 \%$ exists due to both disease-related and patient-related factors. The average treatment cost for triple negative patients amounted to US\$26 923, this is considered to be a more aggressive type of cancer. The overall cost for HER2enriched is driven by the inclusion of Herceptin, thus this subtype is impacted by disease characteristics. Cost variability across molecular classifications is impacted by the severity of the disease, thus disease-related factors are the major drivers of cost.

Conclusions Given the cost challenge in healthcare, the need for greater cost transparency has become imperative. Through our analysis, we generate initial insights into the drivers of cost variability for breast cancer. We found evidence that disease characteristics such as severity and more aggressive cancer forms such as HER2-enriched and triple negative have a significant impact on treatment cost across the different subtypes. Similarly, patient factors such as age and presence of gene mutation contribute to differences in treatment cost variability within molecular subtypes.

\section{INTRODUCTION}

The aim of this study is to analyse the variability in treatment costs for a single disease using the perspective of molecular subtypes
Strengths and limitations of this study

- It is evident that time-driven activity-based costing (TDABC) is a valid and practical method for assessing costs of treatment pathways in breast cancer.

- This study illustrates that TDABC is flexible towards the classification, length and design of treatment pathways.

- This study shows that an adequate level of cost transparency can already be obtained with a minimum set of data.

- The study's focus is costs only and not linked to treatment outcome.

- This study does not allow one to empirically quantify the impact of characteristics on treatment costs.

and several patient-specific and diseasespecific characteristics. Worldwide, it has been documented that healthcare costs are on the rise. ${ }^{1}$ This increase is driven by a number of factors related to ageing population, technological advancements and the current reimbursement system. ${ }^{2}{ }^{3}$ However, in conjunction with these factors it has been noted that significant cost variation exists across hospitals in providing care. ${ }^{4}$ To curb higher expenditure and reduce cost variability, greater understanding of the drivers of variation is warranted. ${ }^{5}$ Using time-driven activity-based costing (TDABC), we aim to quantify the impact of multiple characteristics on the overall cost of treatment. Previous academic studies have drawn attention to identifying particular variables that lead to differences in costs for hospitals. ${ }^{6-10}$ These studies provide evidence that costs can vary based on a number of factors such as hospital structure and physician variables.

We focus on breast cancer as the medical disease to examine the impact of patient and disease factors on the variability of costs. Globally, breast cancer remains the most commonly diagnosed cancer and it is the leading cause 
of death in women. ${ }^{11}$ Breast cancer is considered a heterogeneous disease, and evidence shows that clinically similar tumours often translate into different prognoses and react differently to therapy. ${ }^{12}$ From existing medical literature, we see that patient and disease characteristics play a significant role in the treatment path followed by patients. ${ }^{13}$ The classification of the disease is based on severity, and patients' overall health status impacts the medical decisions taken. ${ }^{14}$ Using the four main molecular subtypes of breast cancer, we aim to quantify the impact of these characteristics on the variability of costs. Our academic study takes place in the breast clinic of a hospital where all patients are treated by a multidisciplinary team (MDT). The MDT is responsible for all medical decisions, and patients are surgically treated by the same oncoplastic surgeon, therefore controlling for hospital and physician variability. To accurately measure costs we use TDABC as the costing technique, which has been proposed by Kaplan and Anderson ${ }^{15}$ and has been used in a growing body of research. ${ }^{1617}$

\section{METHODS}

\section{Overall TDABC model}

Our research was conducted at a breast clinic located in Belgium. All relevant patient and financial data were collected through a research collaboration with a general hospital. Through face-to-face interviews with the physician and medical staff, generic process maps for each step in the intervention path were developed. ${ }^{18}$ A full overview of the treatment steps can be found in online supplementary appendix A. Through designing the process maps, the relevant activities were identified with the corresponding medical personnel, medical equipment and facilities used. Time estimates were based on the average time spent per activity, and the full duration of the activity was covered. The estimates used are based on subjective estimates provided by the medical personnel. All data were collected through interviews with medical staff and through direct observations.

Annual costs for medical equipment and hospital facilities were retrieved from the financial database of the hospital in collaboration with the finance department. All costs used in our analysis are taken from the perspective of the hospital. Salaries include all additional remuneration benefits paid to employees. Given the sensitivity surrounding remuneration, the cost for medical personnel was calculated using figures from a Belgian study published by the Belgian Health Care Knowledge Centre in $2012^{19}$ which is a federal research institution focusing on healthcare research. To provide a more accurate salary figure, an index of $7.2 \%$ was added to account for inflation.

Costs relating to the surgical intervention were made up of medical personnel's salaries, direct medical materials, medical machines and equipment and indirect costs. For our study, we worked with one breast surgeon who works in a standardised way. We had one dedicated operating team made up of one nurse, a surgical trainee and anaesthetist for all surgical interventions, thus we limit any potential variability that may arise from the use of different surgical techniques. Through direct observation of the surgical procedures, full lists were made regarding all the medical instruments used in each type of surgical intervention. The per unit cost of the direct medical materials was provided by the pharmaceutical department of the hospital. These costs represent the direct cost the hospital pays to their suppliers for these materials.

For all medical machines and equipment, the annual cost was made up of the maintenance and depreciation costs for a year. Facilities costs were incorporated into the indirect costs of the departments. The full indirect costs covered depreciation and maintenance costs, financial and general costs, heating, administrations, linen, food, personnel accommodation and other medical costs.

The annual practical capacity was calculated for all resources. The practical capacity used within the hospital for in-house medical staff amounts to 1620 hours/year/ person. This amount takes into account all potential vacation days, clinical days and other administrative breaks. The practical capacity for the medical machines is based on the same available number of hours as in-house medical staff. The more senior medical staff employed as independents have a practical capacity of 2400 hours/ year/person. The cost per unit of resources used is displayed in online supplementary appendix B.

\section{Patient and disease characteristics}

Using the general treatment framework, we consulted medical literature and identified several relevant patient and disease characteristics to analyse how these factors impact the costs through their impact on the treatment path. A full overview of the selected patient and disease factors for this analysis is displayed in table $1 .^{20-22}$ Each treatment step was impacted by the presence of one or more of the listed characteristics. Our analysis focuses on how these factors drive cost differences for patients within and between molecular classifications.

Based on medical guidelines, the starting point for all breast cancer patients is the standard diagnostic process made up of the triple assessment. ${ }^{23}$ This allows physicians to confirm the diagnosis and to assess the tumour biology to define the molecular subtype. Historically, clinicopathological parameters such as tumour size, lymph node involvement and histological grade were used to diagnose breast cancer patients. ${ }^{24}$ However, the advancement of technology has allowed for greater understanding of the molecular biology of the disease. Biomarkers such as Ki-67 proliferation index, estrogen receptor (ER), progesterone receptor (PR) and epidermal growth factor receptor 2 (HER2) are used to classify patients into molecular subtypes. ${ }^{25}$ Several molecular subtypes have been developed using these biomarkers. We focused on the four main subtypes, namely luminal A and B, triple 


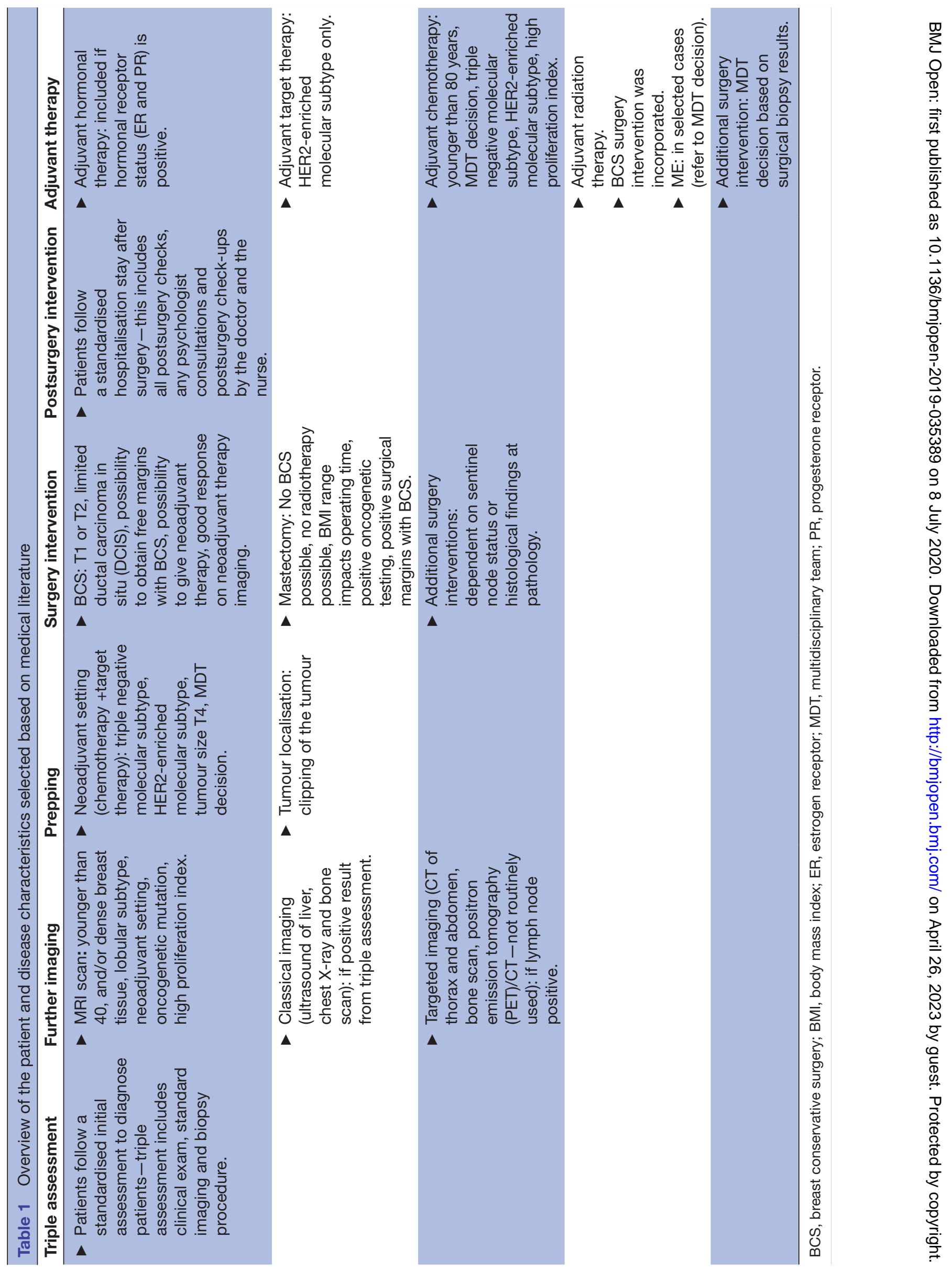


negative and HER2-enriched and use these groupings to stage patients.

Patients classified as luminal A display positive expressions for ER and PR, with the absence of HER2, and have low proliferation and histological grades. ${ }^{20}$ In comparison, luminal B subtypes express lower levels for hormonal receptors (ER and PR) but could express positive levels for HER2.

Luminal B classifications are more aggressive due to their higher histological grade and proliferation levels. ${ }^{2026}$ HER2-enriched cancer displays an overexpression for the HER2 gene pathway, ${ }^{12}$ and the majority of cases express low levels of hormonal genes. ${ }^{27}$ HER2-enriched cancers have high proliferation levels and grade. ${ }^{26}$ The triple negative subtype displays low expressions for all three important receptors, namely ER, PR and HER2, and high grade and proliferation are associated with triple negative tumours. $^{28}$

Based on the molecular subtype and other classical disease parameters, the MDT determines the possible need for neoadjuvant therapy before surgery. Having completed the diagnostic workout and any necessary neoadjuvant therapy, patients are then expected to undergo a surgery intervention compromising of either breast conservative surgery (BCS) or mastectomy (MX). This decision is largely driven by the severity of the disease and in some cases by patient characteristics pertaining to the presence of oncogenetics. Additional interventions are conducted during the surgery such as the sentinel node procedure and the axillary dissection. Inclusion of these measures are driven by positive results of the biopsy testing during surgery. Following surgery, local adjuvant therapy is then administered in the form of radiation therapy. The MDT determines the systemic treatment such as adjuvant chemotherapy, adjuvant hormonal therapy and adjuvant Herceptin. The combination of adjuvant therapy is driven by the molecular subtype of the disease as well as the patient and disease characteristics, such as age of patient at diagnosis and higher tumour grade and size.

The time frame used in our generic treatment pathways varied depending on the inclusion of neoadjuvant therapy, the inclusion of local adjuvant radiation therapy and the type of systemic therapy chosen for the patient. On average the diagnostic workup was performed over a maximum of 2 weeks, including the MRI scan. The surgical intervention was performed 1 week after the full diagnostic workup was complete, unless neoadjuvant treatment was required. For neoadjuvant and adjuvant Herceptin therapy, the costs represent the 18 sessions required resulting in an administration timeline of 12 months. For neoadjuvant and adjuvant chemotherapy, the full costing was used for all required sessions which typically lasts 6 months. The costs for local adjuvant radiation therapy represent the 4-5 weeks standard time period. Based on medical guidelines, regarding the administration of adjuvant hormonal therapy, costs were calculated on either 5-10 years, and this information was provided in the patient files. The cost for endocrine therapy has been included in the adjuvant hormonal therapy amount. For the purpose of our analysis we excluded ovarian suppression costs, and we acknowledge that the inclusion of this activity for premenopausal patients will increase treatment costs as this can either be achieved through a surgical intervention or the administration of GnRH analogues. However, this intervention is required only for a subpopulation of patients; therefore, they were not included in our study.

For our analysis, we used a total of 14 patient files to illustrate the effect of several characteristics identified on the generic treatment path and how those subsequently impacted the overall treatment costs for patients. Our reasoning for selecting these 14 patients was to capture a diverse number of characteristics. The selected patients not only had different characteristics across the four molecular subgroups but they also had differences within the molecular classifications. We excluded patients with extreme comorbidities from our analysis. The presence of comorbidities impacts the decision-making regarding the treatment pathway patients follow and subsequently impacts costs. However, the intersection between severe comorbidities and breast cancer fell beyond the scope of our research. The full treatment cost for each patient was calculated based on the medical interventions and the per unit cost calculated using the TDABC technique. An overview of the patient files and the relevant characteristics of the patients can be found in table 2 .

\section{Patient and public involvement}

Patients had no influence on the research questions or outcomes of this research. No patients were involved in the design of this study. We used blind patient files; therefore, no patient recruitment took place. We included only certain patient and disease characteristics which were made available to us by the physician, and no personal information of patients was used in this study. Given the nature of removing all personal information, there is no requirement to disseminate the information to patients.

\section{RESULTS}

From the costing results, we identified that a significant variation in treatment costs exists between patients within each molecular subtype as well as across molecular subtypes. Typically for luminal A the cost differential amounts to roughly $166 \%$, and the greatest treatment cost amounts to US\$29 780 relative to US\$11 208 for a patient requiring fewer medical activities. The major driver of cost is due to disease-specific characteristics such as the higher tumour grade, tumour size and the presence of extensive lymph node involvement. These characteristics may lead to the inclusion of adjuvant chemotherapy as part of the treatment. When focusing our attention on luminal B, we noticed that patients with identical disease characteristics, such as tumour size and grade, resulted in the same overall treatment 


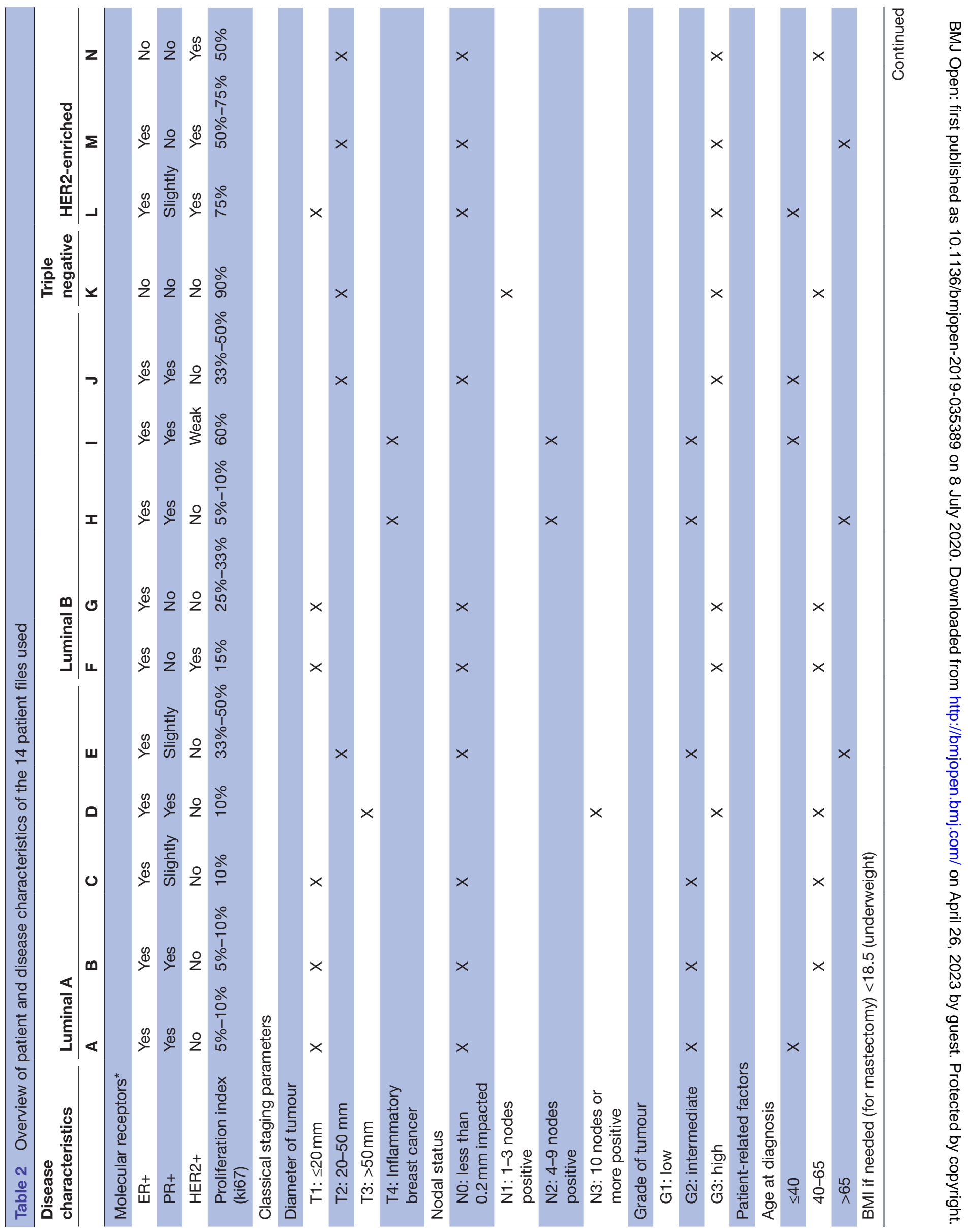




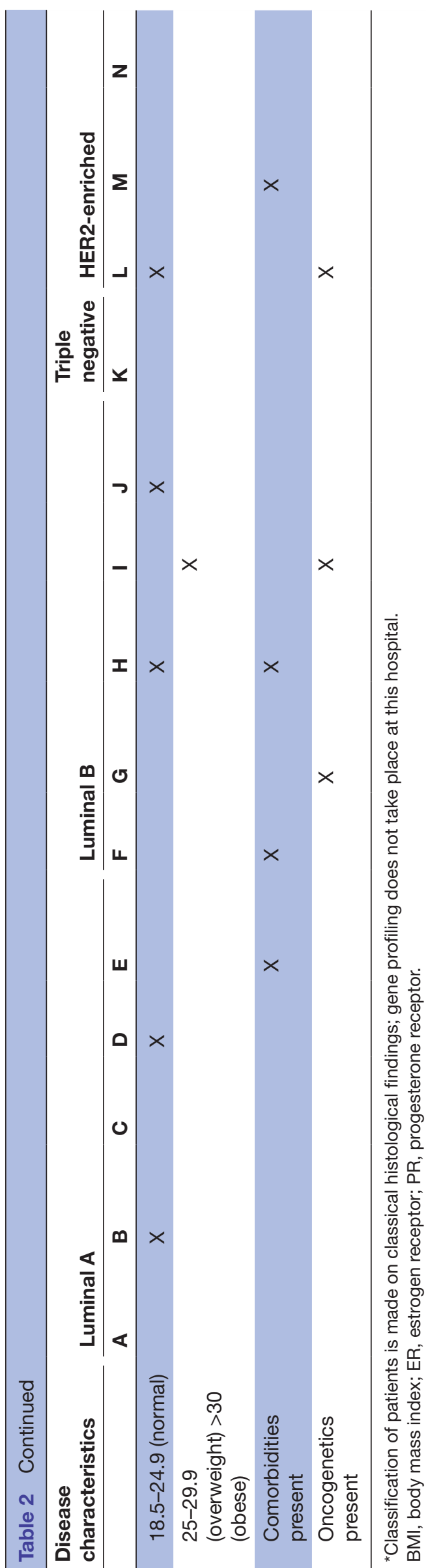

costs, amounting to US $\$ 16335$. However, the treatment costs tend to increase exponentially when the disease characteristics are higher or considered to be more aggressive. Additionally, patient-related factors lead to cost variations for luminal $\mathrm{B}$. We noted that age at diagnosis, particularly patients aged 40 and below, and the presence of oncogenetic factors tend to have higher overall treatment costs due to more aggressive treatment schemes that include neoadjuvant therapy and a combination of surgery interventions and adjuvant therapy. These disease and patient characteristics resulted in a $242 \%$ cost difference whereby treatment costs ranged from US\$13 435 to US\$45 908.

Historically, the triple negative subtype is considered to be a highly aggressive form of cancer. Therefore, costs for this particular subtype are driven by disease characteristics. Based on medical guidelines, neoadjuvant therapy is recommended for this subtype before surgery. Taking this into consideration, the average treatment cost amounts to US\$26 923. The final subtype, HER2-enriched, on average has the highest overall treatment cost due to the inclusion of Herceptin as part of the treatment intervention. Therefore, the characteristics of the disease pertaining to receptor status play an important driver of cost for this subtype. HER2-enriched cancer requires treatment against the HER2 receptor. Additionally, like luminal $\mathrm{B}$, the positive presence of oncogenetics and the age below 40 years impact the cost through the inclusion of neoadjuvant therapy and more aggressive adjuvant therapy schemes.

When considering the costs variability across the molecular subtypes, we notice that the more aggressive classifications like triple negative and HER2-enriched are driven by the disease characteristics. The medical decision-making is heavily influenced by the need to contain the growth of the cancer in the patient. Consequently, the medical interventions needed to treat these types of cancers are more detailed, such as incorporating neoadjuvant therapy into the treatment path, thus by definition leading to cost differences across the classifications. A similar remark can be made about luminal A type cancers that are impacted by the disease characteristics. However, the major motive for costs differences between luminal A, triple negative and HER2-enriched relate to the fact that luminal A type cancer is often considered to be less aggressive. The number of medical interventions required is considerably fewer for luminal A. From our analysis, most luminal A patients did not require extensive adjuvant therapy that generally impacts overall cost. We noted that luminal $\mathrm{B}$ is the most complex classification in that the medical decisions taken for this group can be impacted by both patient and disease-related variables. These equally serve as drivers of cost unlike the other three classifications that are largely impacted by disease characteristics only. The full treatment costs covering all the relevant activities for the 14 patients can be found in table 3 . 


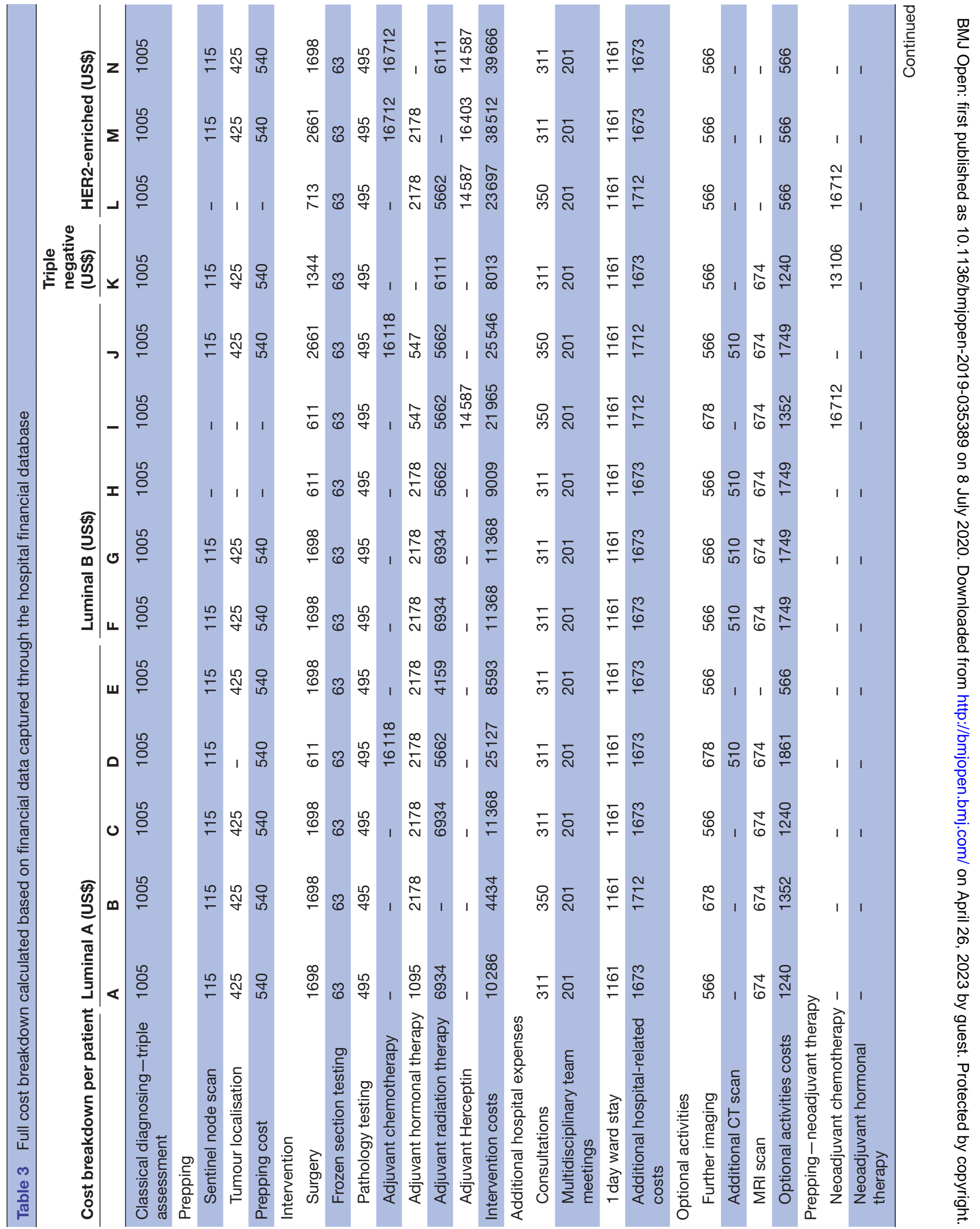




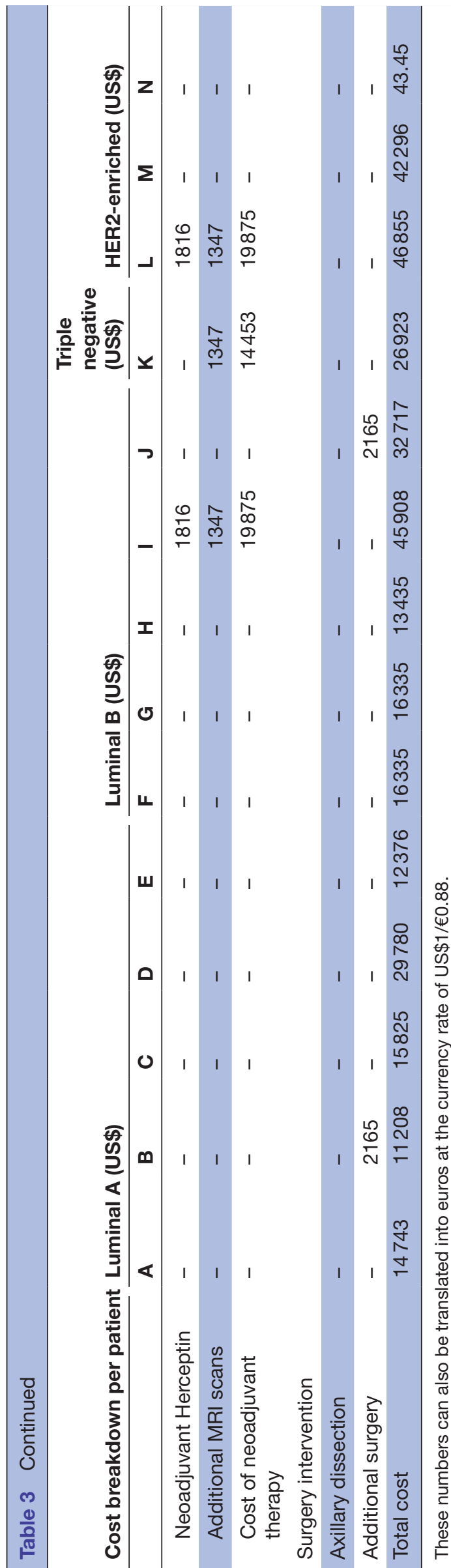

\section{DISCUSSION}

Given the consistent global rise in healthcare costs, an increased need for understanding cost variability and greater cost transparency has been initiated. ${ }^{2}$ Significant value exists when a higher knowledge of cost variability is obtained. Hospitals and management are in a better position to anticipate future spending, make more accurate spending forecasts and make adjustments to their planning and processes. ${ }^{29}$ Additionally, understanding cost fluctuations allows for better measurement of inefficiencies and potentially provides solutions for the current reimbursement system. ${ }^{4}$ In the current state of the sector, little is known about the true cost of treatment for most diseases. Consequently, this lack of knowledge hampers the efforts positioned towards cost reduction and reimbursement changes. Through our analysis, we focus on understanding how treatment costs vary as a result of several patient and disease characteristics. This perspective enables medical providers and physicians to predict the potential costs of treatment upfront, which is not currently being done. ${ }^{30}$

When shifting our focus onto the particular molecular subtypes, we are able to identify specific characteristics contributing to the fluctuations in treatment costs. When looking at the luminal A subgroup, we noticed that disease characteristics impacted the treatment path decision. Luminal A breast cancer is considered to have a less aggressive tumour biology, in comparison with the other molecular subtypes, and has a better prognosis. However, when looking at the patient files we noticed that even within luminal A some patients may have more aggressive cancers. For this particular subgroup, the major driver for decision-making boils down to the characteristics of the disease. Two patients with similar age and patient-related factors have major cost variability. We noted that higher tumour grade, a larger tumour size and the presence of extensive nodal involvement may require the inclusion of adjuvant chemotherapy for treatment, as higher levels of these factors imply that patients are at greater risk. ${ }^{31}$

For the luminal B subtype, both patient and diseaserelated factors could potentially play a role on cost variation. In the one instance, patients $F$ and $G$ had vastly similar disease-specific characteristics such as tumour grade, tumour size and nodal status, thus requiring identical treatment interventions resulting in treatment costs totalling roughly US $\$ 16335$. Patients $\mathrm{H}$ and I had comparable disease characteristics but major differences related to patient characteristics. Patient I was below the age of 40 at the time of diagnosis and tested positive for both the HER2 receptor and the BRC2 mutation gene. The presence of BRC2 potentially translates into an increased chance of locoregional relapse and/or an increase of contralateral breast cancer. These specific patient factors may lead to the inclusion of neoadjuvant therapy (chemotherapy and Herceptin) and may influence the type of surgery, in some cases, with or without prophylactic MX instead of BCS. The 
inclusion of neoadjuvant and adjuvant therapy potentially increases the overall treatment price by approximately US\$34 000 .

Both HER2-enriched and triple negative are classified as more aggressive types of cancer. The total costs are largely driven by the increased combination of interventions needed. The tumour grade and proliferation index for these subtypes are generally higher. Given the name, HER2-enriched patients test positive for the HER2 receptor, requiring either neoadjuvant Herceptin therapy or adjuvant Herceptin therapy. The inclusion of Herceptin into the proposed treatment plan has a dramatic increase in the total costing. The full administration of Herceptin increases the treatment cost by approximately US $\$ 16403$ per patient. Therefore, this particular molecular subtype carries an enormous cost burden.

In comparison, triple negative subtypes display negative expression for all receptors. However, both patient characteristics like age and disease characteristics impact the cost based on the treatment decision. High tumour grades and high proliferation grades require more aggressive interventions increasing the cost through the need for treatment regimens which include chemotherapy. The full costing for chemotherapy per patient averages around US $\$ 16415$. For the triple negative patient, the inclusion of adjuvant chemotherapy is driven by the high proliferation levels and the grade of the tumour requiring more aggressive treatment.

When shifting our focus to other academic studies, we find one study within breast cancer which has calculated and analysed costs of a particular treatment step, like the radiation therapy, in the entire treatment process. ${ }^{17}$ Some studies have used conventional breast cancer staging to calculate costs for each stage using the average cost in hospitals. ${ }^{32}$ Additionally, specific medical studies have looked at linking molecular classifications to the prognosis of cancer. ${ }^{33}$ Other studies focusing on cost variability have focused mainly on hospital-related characteristics and the impact on average costs. These studies found that characteristics such as the type of hospital structure (public vs private), the level of innovation and medical technology in the institution, the case load of surgeons and discharge dispositions influence costs. $^{6-9}$

As part of the value era one of the key reform initiatives to depart from the current fee-for-service reimbursement system is the introduction of bundled payments, whereby the full episode of care is financed. The initiation of bundled payments highlights the need for healthcare providers to predict which patients are at risk of higher treatment costs for a disease. ${ }^{34}$ To achieve this, we have to turn our attention to the factors that drive variability. We provide initial insights into the average treatment costs for the molecular subtypes highlighting the cost impact of several disease and patient characteristics on the treatment pathway. By shifting the focus to analysing costs through the perspective of molecular subtypes and identifying the impact of several characteristics on cost, we aim to provide an alternative perspective into the way bundled payments could potentially be introduced.

In addition to generating an alternative approach for dealing with the change in the reimbursement system, we believe that this method could form the basis for other medical domains for future academic research. We chose to analyse patient and disease characteristics within early-stage breast cancer because of the disease's standardised nature. All patients undergo triple assessments for diagnosing, followed by a generic neoadjuvant and adjuvant treatment pathway. However, we recognise that the perspective of understanding cost variability could be applied to other disease domains. Insights could be generated in disease areas which follow a standard diagnosing path, ones with minor surgical intervention and a generic treatment path postsurgery. An alternative oncological domain that could potentially be interesting to explore would be colon cancer that has standardised biopsy interventions. The field of dermatology serves as an interesting non-oncological domain as the treatment pathology is highly standardised for patients. Additionally, other medical domains that follow a similar disease classification like the molecular subtypes found in breast cancer serve as another interesting medical disease to which this approach could potentially be applied.

Using the generic treatment pathway developed, we provide evidence that significant cost variability exists across molecular subtypes and within molecular subtypes. We provide evidence that patient and diseaserelated factors impact the treatment path and highlight the impact on costs. However, one limitation of our study relates to the fact that limited generalisations can be made given the limited patient data used. For the purpose of our analysis, we selected 14 patient files to provide initial results that cost variability does exist as a result of patient and disease characteristics within the molecular subtypes. We believe that an extension to our research could focus on understanding how and to what extent these characteristics drive or impact cost variability through an empirical analysis using a larger sample size. Furthermore, a larger sample size allows one to explore the statistical impact of several characteristics on driving cost differences. This serves as an interesting avenue for research focusing on understanding cost variability in disease settings.

Another potential extension of this research is to link costs to the quality of care provided to generate insights into cost-effectiveness. With the push towards a value system, studies that focus on both costs and outcomes have become imperative. With the limitation surrounding the availability of quality data, we were unable to provide relevant academic findings for how this alternative perspective could impact the outcomes achieved for treating patients with breast cancer. Therefore, we recognise a need for future research that 
focuses on costs and their link to quality. By looking at costs and the impact costs have on quality, we move into a realm of being able to provide insights into costeffectiveness strategies.

In conclusion, specific patient and disease characteristics play a major role in the treatment interventions and pathway for patients. Each molecular subtype is impacted by the range of patient and disease-related factors. These factors impact the overall cost of treatment within the four major molecular subtypes as well as across the molecular subtypes. Significant cost differences exist across the subtypes due to the severity of the disease, more aggressive forms such as HER2-enriched and triple negative require increased medical interventions leading to higher overall costs. Cost differences within molecular subtypes are driven in part by disease characteristics, but also heavily influenced by patient factors. By using the medical focus of the disease and linking it to costs and understanding the drivers of cost, we move one step closer to making more sound cost reduction decisions.

Acknowledgements We would like to thank Sabine Verhofstadt (CF0) \& Marc Muylaert (Finance department) from Sint-Blasius for all their help in the collection of data, as well as the reviewers for their feedback and suggestions.

Contributors ER is lead author of this manuscript. She was responsible for the full collection of data used within the manuscript. Additionally, she performed the analysis for the study and was responsible for writing the original draft of the article. BC is a coauthor. He was responsible for the conception and design of the analysis. He played a key role in the development of the methodology and was part of the team who acquired the funds for the study. JD is listed as coauthor. He played an important role in obtaining and providing all the data used for the study. He contributed to the methodology applied from a medical perspective. He assisted ER in the investigation of the molecular subtype classification and in the selection of the key patient-related and disease-related characteristics. FR is listed as coauthor for this manuscript. He played a key role in the design and conception of the analysis. Additionally, he contributed to the development of the methodology applied in the study. He played a key role in the acquisition of the funding needed to conduct the research.

Funding Co-funded by an unconditional grant provided by Xperthis in Belgium, but no conflict of interest to be reported.

Competing interests None declared.

Patient and public involvement Patients and/or the public were not involved in the design, or conduct, or reporting, or dissemination plans of this research.

Patient consent for publication Not required.

Provenance and peer review Not commissioned; externally peer reviewed.

Data availability statement Data are available upon reasonable request. All data pertaining to the costing information can be shared and reused for future research. All data relating to the patients characteristics can be shared and reused for other studies as no personal information was used. All data can be made available at request by the corresponding author.

Open access This is an open access article distributed in accordance with the Creative Commons Attribution Non Commercial (CC BY-NC 4.0) license, which permits others to distribute, remix, adapt, build upon this work non-commercially, and license their derivative works on different terms, provided the original work is properly cited, appropriate credit is given, any changes made indicated, and the use is non-commercial. See: http://creativecommons.org/licenses/by-nc/4.0/.

\section{ORCID iD}

Erin Roman http://orcid.org/0000-0003-3194-3231

\section{REFERENCES}

1 Porter ME, Lee TH. The strategy that will fix healthcare. Harv Bus Rev 2013;1277:1-19.
2 Kaplan RS, Porter ME. How to solve the cost crisis in health care. Harv Bus Rev 2011:89:2011-3.

3 Kaplan RS, Witkowski ML. Better accounting transforms health care delivery. Account Horiz 2014;28:365-83.

4 Dormont B, Milcent C. The sources of hospital cost variability. Health Econ 2004;13:927-39.

5 Houÿez F, Tessier L, Synodinos D. Health care cost-containment measures in the context of the economic crisis: impact analysis. Orphanet J Rare Dis 2014;9:021.

6 Childers CP, Maggard-Gibbons M. Understanding costs of care in the operating room. JAMA Surg 2018;153:e176233.

7 Robinson JC, Pozen A, Tseng S, et al. Variability in costs associated with total hip and knee replacement implants. J Bone Joint Surg Am 2012;94:1693-8.

8 Kilic A, Shah AS, Conte JV, et al. Understanding variability in hospital-specific costs of coronary artery bypass grafting represents an opportunity for standardizing care and improving resource use. $J$ Thorac Cardiovasc Surg 2014;147:109-16.

9 Tomek IM, Sabel AL, Froimson MI, et al. A collaborative of leading health systems finds wide variations in total knee replacement delivery and takes steps to improve value. Health Aff 2012;31:1329-38.

10 Martin JA, Mayhew CR, Morris AJ, et al. Using time-driven activitybased costing as a key component of the value platform: a pilot analysis of colonoscopy, aortic valve replacement and carpal tunnel release procedures. J Clin Med Res 2018;10:314-20.

11 Bray F, Ferlay J, Soerjomataram I, et al. Global cancer statistics 2018: GLOBOCAN estimates of incidence and mortality worldwide for 36 cancers in 185 countries. CA Cancer J Clin 2018;68:394-424.

12 Rouzier R, Perou CM, Symmans WF, et al. Breast cancer molecular subtypes respond differently to preoperative chemotherapy. Clin Cancer Res 2005:11:5678-85.

13 Coates AS, Winer EP, Goldhirsch A, et al. Tailoring therapiesimproving the management of early breast cancer: St Gallen International Expert Consensus on the Primary Therapy of Early Breast Cancer 2015. Ann Oncol 2015;26:1533-46.

14 Cardoso F, van't Veer LJ, Bogaerts J, et al. 70-Gene signature as an aid to treatment decisions in early-stage breast cancer. $N$ Engl J Med 2016;375:717-29.

15 Kaplan RS, Anderson SR. Time-Driven Activity-Based Costing: A Simpler and More Powerful Path to Higher Profits. Boston: Massachusetts: Harvard Business School Publishing Corporation;, 2007.

16 RYC T, Met-Domestici M, Zhou K, et al. Using quality improvement methods and time-driven activity-based costing to improve valuebased cancer care delivery at a cancer genetics clinic. J Oncol Pract 2016.

17 Schutzer ME, Arthur DW, Anscher MS. Time-driven activity-based costing: a comparative cost analysis of whole-breast radiotherapy versus balloon-based brachytherapy in the management of earlystage breast cancer. J Oncol Pract 2016;12:e584-93.

18 Keel G, Savage C, Rafiq M, et al. Time-driven activity-based costing in health care: a systematic review of the literature. Health Policy 2017;121:755-63.

19 Swartenbroekx N, Obyn C, Guillaume P, et al. KCE Reports $178 \mathrm{~A}$ - Handleiding Voor Op-Kosten-Gebaseerde Prijsbepaling van Ziekenhuisinterventies. Belgium: Belgian Health Care Knowledge Centre, 2012: 128. /nl/handleiding-voor-op-kosten-gebaseerdeprijsbepaling-van-ziekenhuisinterventies.

20 Curigliano G, Burstein HJ, Winer EP, et al. De-escalating and escalating treatments for early-stage breast cancer: the St. Gallen international expert consensus conference on the primary therapy of early breast cancer 2017. Ann Oncol 2017;28:1700-12.

21 Paluch-Shimon S, Pagani O, Partridge AH, et al. ESO-ESMO 3rd international consensus guidelines for breast cancer in young women (BCY3). The Breast 2017;35:203-17.

22 Henry NL, Somerfield MR, Abramson VG, et al. Role of patient and disease factors in adjuvant systemic therapy decision making for early-stage, operable breast cancer: American Society of clinical oncology endorsement of cancer care Ontario guideline recommendations. J Clin Oncol 2016;34:2303-11.

23 Senkus E, Kyriakides S, Ohno S, et al. Primary breast cancer: ESMO clinical practice guidelines for diagnosis, treatment and follow-up. Ann Oncol 2015;26 Suppl 5:v8-30.

24 Malhotra GK, Zhao X, Band H, et al. Histological, molecular and functional subtypes of breast cancers. Cancer Biol Ther 2010;10:955-60.

25 Eroles P, Bosch A, Pérez-Fidalgo JA, et al. Molecular biology in breast cancer: intrinsic subtypes and signaling pathways. Cancer Treat Rev 2012;38:698-707. 
26 Ades F, Zardavas D, Bozovic-Spasojevic I, et al. Luminal B breast cancer: molecular characterization, clinical management, and future perspectives. J Clin Oncol 2014;32:2794-803.

27 Prat A, Pascual T, Adamo B. Intrinsic molecular subtypes of HER2+ breast cancer. Oncotarget 2017;8:73362-3.

28 Nielsen TO, Hsu FD, Jensen K, et al. Immunohistochemical and clinical characterization of the basal-like subtype of invasive breast carcinoma. Clin Cancer Res 2004;10:5367-74.

29 Dieleman JL, Campbell M, Chapin A, et al. Future and potential spending on health 2015-40: development assistance for health, and government, prepaid private, and out-of-pocket health spending in 184 countries. The Lancet 2017;389:2005-30.

30 Lee VS, Kawamoto K, Hess R, et al. Implementation of a value-driven outcomes program to identify high variability in clinical costs and outcomes and association with reduced cost and improved quality. JAMA 2016;316:1061-72.

31 Wildiers H, Denys H, Fontaine C, et al. Adjuvant and neoadjuvant chemotherapy regimens in breast cancer: summary from the BSMO breast cancer Task force meeting 2014;8:9.

32 Mittmann N, Porter JM, Rangrej J, et al. Health system costs for stage-specific breast cancer: a population-based approach. Curr Oncol 2014;21:281-93.

33 Taherian-Fard A, Srihari S, Ragan MA. Breast cancer classification: linking molecular mechanisms to disease prognosis. Brief Bioinform 2015;16:461-74.

34 Medbery RL, Perez SD, Force SD, et al. Video-assisted thoracic surgery lobectomy cost variability: implications for a bundled payment era. Ann Thorac Surg 2014;97:1686-93. 